\title{
Treatment Options for Pin Site Infection during Kirschner Wires in Elective Forefoot Surgery
}

\author{
Ismail Mohamed Hussein, Lei Wang, Bin Yu* \\ Department of Orthopedic and Traumatology, Guangdong Provincial Key Laboratory of Bone and Cartilage Regenerative \\ Medicine, Nanfang Hospital, Southern Medical University, Guangzhou, China \\ Email: *yubin@smu.edu.cn
}

How to cite this paper: Hussein, I.M. Wang, L. and Yu, B. (2021) Treatment Options for Pin Site Infection during Kirschner Wires in Elective Forefoot Surgery. Open Journal of Orthopedics, 11, 47-66. https://doi.org/10.4236/ojo.2021.112006

Received: January 10, 2021

Accepted: February 23, 2021

Published: February 26, 2021

Copyright (c) 2021 by author(s) and Scientific Research Publishing Inc. This work is licensed under the Creative Commons Attribution International License (CC BY 4.0).

http://creativecommons.org/licenses/by/4.0/

(c) (i) Open Access

\begin{abstract}
The Kirschner wires (K-wires) are stiff and straight wires made of smooth stainless steel. These are used in surgery to aid in repairing the process of a fractured bone. In forefoot elective surgeries, these pins are mostly used for fixation of the phalangeal or metatarsal area. These wires are usually either buried or exposed, The exposed wires do not require another operation to remove them and are generally preferred. In using the forefoot surgery history of a single surgeon which was taken for 3 years. The purpose of this study is to evaluate the occurrence of infections following elective surgeries of the forefoot that were fixed using k-pins and to indicate an effective treatment option for resolving the infection. The inclusion criteria set for this study was to remove any patient having any disease except for diabetes. Moreover, the age limit was set to be 18 - 44, making a final sample of 50 people, prescribed prophylactic antibiotics or early removal of k-pins, where necessary. The researcher recorded demographic and biological data from the patients such as age, BMI, and smoking status were recorded. The researcher conducted a Univariate analysis via a Chi-square test. 50 Patients ( $82 \mathrm{~K}$-wires) were analyzed. Results showed that surgery type, BMI, and smoking were three variables that affect the infection growth significantly while diabetes and type of cleaning disinfectant had marginal effects. Further study is required to clarify the best treatments in this area, as the treatment option antibiotics have nearly similar results.
\end{abstract}

\section{Keywords}

Kirschner Wires, Complications, Risk Factors, Disinfectant, Forefoot Surgery, Infection Risk, Treatment Options

\section{Background}

The Kirchner wire (K-wire) is a device commonly utilized for temporary fixation 
in the forefoot [1]. Majorly due to their complex articulations, smaller size, and anatomic location, metatarsals, and phalanges often require K-wire fixation when the screw and plate are not optimal or feasible. During proper alignment and placement, K-wires can significantly remain in place for up to 6 weeks that helps to make a bony correction to heal in the desired position [2]. But sometimes, the negligence in implementing these $\mathrm{K}$-wires causes many pin-site infections based problems that are directly associated with the external fixation treatment. This infection mostly begins at the level of the skin; if it is determined early, then can be treated easily. But if left untreated then they may progress to the deeper soft tissues and possibly affected the bone [3]. There are many symptoms of pin site infection like skin redness, hardness or swelling of the skin, warmer and excessive pain at pin site. Its drainage is yellow, thick, green, or smelling. Those patients who are in a chronic situation of fracture may face such issues due to growing biofilms and tracking down Kirchner wires [4]. Such kinds of Pin site infection issues due to K-wires are mostly developed in elective forefoot surgery which is defined as operations that are scheduled and performed in advance. Such medical surgeries are not for a medical emergency and may be done for cosmetic/medical reasons to relieve pain and discomfort [5]. The most common elective foot surgeries are hammertoes, bunion removal, arthritis of ankle \& foot, and a plethora of others. China, a developing nation, is famous because of its advanced technology-oriented initiatives in every field of life and business, also faced such infection issues due to K-wires among surgical patients [6].

\section{Kirchner Wires Treatment in Surgeries}

Kirchner wires are such thick metallic pins or wires that are mostly used to stabilize the bone fragments which majorly drilled through the bone to significantly drill through bone to hold fragments. In previous researches, the majority of scholars majorly worked on considering the implications of K-wires inefficient treatment of surgeries. Like Gregory Pace along with others majorly worked on considering the $\mathrm{K}$-wire and its antibiotic use in forefoot surgery by stating that an ankle and foot practice-based population has higher rates of comorbidities that result in infection. They assessed the national trends regarding postoperative prophylactic antibiotic therapy usage in patients related to ankle and foot surgery treated with percutaneous K-wires. According to their outcomes, non-diabetic patients must place on postoperative antibiotics for an average of 9.4 days with an average duration of Kirschner wire fixation of 35.1 days, and diabetic patients with neuropathy would place on postoperative antibiotics of 14.5 days with an average duration of K-wires fixation of 36.7 days. It means diabetic patients' situation is riskier for infection than non-diabetic patients' [7]. After this, James McKenzie and others (2019) stated that K-wires could be placed for weeks postoperatively and at complication's risk. They developed a single-surgeons' retrospective chart review of forefoot surgeries and concluded 
that there were 35 pin site infections for a rate of $1.74 \%$. According to them, more risky situations of pin site infection occurred among male sex, excessive smoking, body mass index, and situation pins [8]. In the Journal of Foot and Ankle Surgery, Mangwani and others majorly worked on examining the prospective randomized controlled trials in order to inspect the occurrence of local infection for lesser toe fusion surgery. According to their outcomes, no clear evidence had been seen to support the prophylactic antibodies' use in routine surgery that required percutaneous K-wire fixation because very few patients showed such signs of infection [9]. In 2018, Syed Dil Bagh Ali Shah and others majorly worked on assessing the outcomes of ulna fracture fixation and the pediatric unstable radius with intramedullary K-wires. They majorly treated with open reduction and internal fixation with Kirchner wires where the students assessed through the range of forearm, wrist and elbow, and concluded that such open reduction and $\mathrm{K}$-wires fixation caused a good to an excellent outcome for the unstable forearm fractures in children [1]. According to Junjun Tang and others (2019), the double Kirschner wires are used for the efficient treatment of old bony mallet finger. In the Journal of Orthopaedic Surgery and Research, they majorly worked to presenting an advanced method of open reduction and compression with the double Kirshner wires (K-wires) in the efficient treatment of old bony mallet finger. In order to fulfill this aim, they majorly conducted the retrospective analysis of related patients and measured their range of motion (ROM) of DIP joints. According to their outcomes, seventeen patients were followed up for 8 months, and the width of the avulsion fracture block was accounted for $25 \%-62 \%$ of the distal phalanx's articular surface. All-around $70.6 \%$ of patients had anterior dislocation of an interphalangeal joint with the healing time up to $7.6 \pm 2.1$ (5 - 13) weeks. In their K-wires based surgical outcomes, only one patient developed mild pain after this surgery whose VAS score was 3. Overall, they concluded that open reduction and reduction along with double K-wires is more appropriate in treating the old bony mallet finger [10]. Israr Ahmad and others also considered the Kirchner wire for the fixation of unstable radius-ulna fractures especially in Children's cases where they treated with internal fixation and open reduction of both forearm bones with K-wires. They utilized the retrograde method under the tourniquet and anesthesia to control the fractures. In such surgical treatment, K-wires were removed after healing of fractures. In their results, the average time for the radiological and clinical union was 9.2 weeks in which the interval between the first surgery and the K-wires removal was 3.2 months, and after this, 18 excellent assessments had shown good and two fair results [11]. In the international journal of orthopedics sciences, the related scholars also considered the usage of K-wires to rescue the supracondylar fracture among children in which they considered the humerus or upper arm bone injury that was frequently caused by a fall on direct blow to the elbow or an outstretched elbow. In that surgical study, they majorly dealt with closed removal of fracture and the K-wire based percutaneous fixation under the 
C-arm guidance. According to their statistical outcomes, it becomes concluded that percutaneous fixation and closed reduction by using K-wires of humerus' displayed supracondylar fractures would be safe, cheap, simple, and the effective way to reduce the hospital stay by developing such treatment which has relatively fewer complications [12]. In addition to this, other scholars also worked on a related topic by evaluating the outcome of phalangeal fractures based surgery along with K-wires in the Hue Central Hospital. According to Nugyen Quoc Linh, the complication of $\mathrm{K}$-wires fixation shown a low rate in postoperative recovery which means this technique is safe, effective, and inexpensive with low morbidity. In addition to this, many researchers had made authentic researches to consider the Kirschner wire fixation as a treatment of medial malleolar fractures and cavitary-type scaphoid nonunions [13] [14] [15]. All of these scholars majorly stated that $\mathrm{K}$-wires with auto compression effect directly caused a small fragment fixation and mostly generated satisfactory results in the internal fixation and open reduction of fractured bones and joints. Also, Kirchner Wire as a temporary intramedullary fixation was considered by Jianxin Xie and others in their research journal in order to combine it with locking anatomical plate for the efficient treatment of comminuted clavicular fractures. These scholars majorly considered 112 related patients whose complication rate and fracture healing time was significantly shorter, and no significant difference had been observed in shoulder function scoring between groups. They concluded that $\mathrm{K}$-wire with a temporary intramedullary fixation for locking the anatomical plate plays as a major treatment choice for the disintegrated claviculated fractures because of the less intraoperative blood loss, quicker fracture healing, easier reduction of operation, shorter operation time, and the lower postoperative complication rate [16]. In previous literature, there was also an in-depth study on considering the side effects of $\mathrm{K}$-wires fixation for the distal radius fractures. Like Rick Tosti and others majorly worked on considering the Kirschner wire infections in pediatric orthopedic surgery by describing factors to better prevent and treat infectious complications of smooth wires. They specifically considered the retrospective review to identify all patients who were majorly hospitalized for Kirschner wire infection from 1995 to 2012. According to their outcomes, Kirschner wire infected twelve patients in which three had lateral humeral condylar fractures, five had supracondylar fractures, and the remaining had a great toe open fracture, a distal tibia physeal fracture, an elective osteotomy for hallux valgus, and a distal radius fracture. While the patient was exposed to a soft-tissue abscess in four cases, cellulitis in three cases, toxic shock syndrome in one case, and osteomyelitis in four cases. They concluded that reoperation was required for five patients with septic arthritis, abscess, or osteomyelitis. It shows that many complications had been occurred due to K-wires especially among five major patients in the form of joint destruction, loss of range of motion, catheter migration, wound breakdown, and toxic shock syndrome. They also proposed that improved postoperative education may reduce the risk of infected K-wires 
in severe surgery [17]. Palaniappan Lakshmanan and Varun Dixit majorly worked on considering the infection rate among 43 patients who experienced the $\mathrm{K}$-wires for the distant radius fractures. In the selected participants, each fracture was majorly fixed with 2 to 3 wires of $1.6 \mathrm{~mm}$ in diameter. In their study, Kirschner wires were left bulging through the patient skin for easy removal with the ends bent outside the skin to overcome the migration. In the end, wounds were cleaned and dressed in a plaster-of-Paris and gauze. According to their outcomes, nine $(21 \%)$ of patients developed pin tract infection, and only three patients passed through the removal of Kirschner wires at week 3 [18]. The somehow similar research has been conducted by other scholars in the Journal of Hand Surgery, in which they majorly explored the Kirschner wire-based pin tract infection rate by making a randomized controlled trial between the buried and percutaneous wires. At the end, they concluded that the skin infection rate is excessively increased due to implementing the percutaneous $\mathrm{K}$-wires that directly buried deep to skin with isolated distal radical fractures [19].

\section{Pin Site Infections in Elective Forefoot Surgery}

In K-wires-based surgery, many side effects have occurred and pin site infection is the major one that created a major barrier in the productive outcomes of elective surgery. In previous researches, many scholars also worked on exploring such infection in elective forefoot surgery. Like in 2017, Gregory Pace and others majorly worked on exploring the side effects of antibiotics use and Kirschner wire fixation in the forefoot surgery by conducting a national surgery. According to them, the infection rate is much higher in the typical foot and ankle practices based surgeries. They majorly assessed the postoperative prophylactic antibiotic therapy among patients by specifically identified the 112 physicians for surgery purposes. After critically evaluate the surgical outcomes, they concluded that those diabetic patients who were treated with percutaneous Kirschner wires on postoperative antibiotic prophylaxis were more shown the range of infections on the skin [7]. In 2020, informative research is conducted by Alexandria Case and others by specifically considering the variation among surgeons regarding preoperative antibiotics' usage in the percutaneous pinning process of the upper extremity in the pediatric population. According to their outcomes, $81 \%$ of 295 respondents regularly ordered preoperative antibiotics for all percutaneous pinning procedures of the upper extremity by surgeons, and very few surgeons feel preoperative antibiotics as necessary [20]. Another medical research was conducted by Rachel Albright and Adam Fleischer in the same year by focusing on hammertoe surgery as the most popular elective forefoot surgery performed. According to them, ankle and foot surgeons faced various options for hammertoe surgery with a respected benefit/risk profile [21]. In the Journal of Techniques in Foot and Ankle Surgery, medical researchers considered the deformity of lesser and digits metatarsals as common surgical issues interlinked with ankle and foot surgeries where an elective operation is the only straightforward way to 
resolve the issue. They found different risk factors of surgical complications like inadequate surgical procedure, complications related to surgical procedure, choosing an inadequate surgical technique, and underestimation of unrealistic expectation and inherent risk [22]. In 2018, Ravi Krishan Modha and others worked on a systematic review of literature related to antibiotic prophylaxis in ankle and foot surgery by utilizing CINHAL, MEDLINE, EMBASE, and Cochrane library from 1990-2018. According to their SSI rates based outcomes in a systematic review, $0 \%$ to $9.4 \%$ of overall postoperative infections encountered after ankle and foot surgery [23]. After this, Kevin Tootle with others also worked on the related surgical topic by focusing on its treatment of severe hallux abductovalgus with the dislocation of the first metatarsophalangeal joint. They significantly derived a surgical technique that may use to significantly address the challenging situation while minimizing complications' likelihood [24]. In the Journal of Foot and Ankle Surgery, Mangwani, and others (2017) majorly worked on considering the incident of local infection for the lesser toe fusion surgery under the influence of prospective randomized controlled trial. In this authentic research study, these scholars majorly considered 100 patients and divided them into two groups like the first one was caring for prophylactic antibiotics and the second one didn't have prophylactic antibiotics. According to their outcomes, the overall infection rate was $4 \%$ like 3 patients from one group and 1 patient to another group developed a sign of infection because of the existence of oral antibiotics' treatment. While no evidence had been seen to support the usage of prophylactic antibiotics in the regular toe surgery that majorly required the K-wire insertion. But, the inappropriate use of antibiotics caused a major contribution to enhancing the infection-based antibiotic resistance that adds to healthcare costs [9]. Also, Brent Wise and others (2019) considered the predictive score to efficiently determine the surgical site infection-related risks after making an orthopedic trauma surgery. Within their study, they majorly designed the retrospective cohort study by setting a level one trauma center. They majorly evaluated the twenty-seven factors associated with the postoperative infection along with multiple and bivariate logistic regression analysis that directly help to build a prediction model. Their major aim was to consider the postoperative infection by focusing on different predictors about patients named as diabetes, obesity mass index, alcohol abuse, male sex, fracture region, methicillin-resistant based Staphylococcus aureus nasal swab testing, and the Gustilo-Anderson type III based open fracture. At the end, they concluded that the infection rate was much higher among the fractured patients because of the proposed postoperative infection prediction model which also helped to estimate the percentage of infection risk before the fixation [25]. In 2020, Javier Aragon-Sanchez majorly considered diabetic patients' foot surgery i.e. osteomyelitis and prophylactic surgery. For that aim, they majorly obtained a long relapse-free period and then classified the diabetic foot surgery into four types. According to their clinical outcomes, the elective surgery showed the procedures which per- 
formed on patients with protective sensation in order to improve function or eliminate pain, while the prophylactic surgery directly involved the process performed on patients with the absent protection sensation. After this, the emergency surgery majorly covered the process which is performed with the aim of reducing the life-threatening infection, and the curative surgery shown the procedure performed on open wound based patients with the focus on reducing the recurrence risk and promoting healing's goal [26]. In the forefoot surgeries, the hammertoes based surgery is considered as the most common surgery in which the imbalance situation is created between stronger extrinsic muscles and weak intrinsic muscles that are surrounded by metatarsophalangeal joints of lower digits. Miranda Goransson and Dustin Constant (2020) majorly worked on this concept by specifically worked on its etiology, epidemiology, physical \& history, evaluation, management/treatment, prognosis, diverse diagnosis, complications, deterrence, and patient education. According to their informative research studies, within such hammertoes based electric surgeries, many issues have arisen like avascular necrosis, nonunion, infection, malunion, malalignment, avascular necrosis, mallet toe, stiffness, pain, numbness, PIP joint instability, chronic edema, recurrent deformity and vascular impairment [27]. In the same year, another authentic research was conducted by Rachel Albright and others in the international journal of foot and ankle by stating that there are many risk factors associated with hammertoe surgery failure that result in the increasing range of revision surgery. These scholars concluded that many risk factors like infection are directly associated with preoperative hammertoe surgeries [28]. According to Mordical Atinga and others who majorly worked on making a prospective review of medium-term outcomes under the interposition arthroplasty for the hammertoe deformity correction, and selected the related patients for this research study. According to their outcomes, there was a high satisfaction level that results in pain relief. Also, no complication i.e. infection or chronic pain had been seen with early recurrences which shows good and excellent results with this forefoot surgical tactic by allowing early mobilization, pain relief, and low risk of infections [29]. Also, important and major research work had been conducted on elective forefoot surgery and its complications based perspective. Like Cancienne Jourdan and related ones majorly considered the hemoglobin $A_{1 c}$ factor as a predictor of postoperative infection in the elective forefoot surgery. According to their authentic research-based outcomes, the threat of postoperative SSI was much high when the perioperative $\mathrm{HbA}_{1 \mathrm{c}}$ increased. While their ROC analysis determined that a high perioperative $\mathrm{HbA}_{1 \mathrm{c}}$ more than 7.5 $\mathrm{mg} / \mathrm{dL}$ directly served as a threshold for significantly increased the risk of postoperative SSI considering forefoot surgery [5]. While, Rohit Samuel with the related scholars' team also explored the postoperative pain in forefoot surgery by considering the efficacy of combined ankle and popliteal blocks. They considered the elective osseous surgical procedure on the forefoot surgery, and their outcomes depicted that patients with combined popliteal and ankle block 
showed significantly less pain. So, at the end, they concluded that popliteal block in conjunction with the ankle block directly provides a significantly better relief as compared to ankle block in patients undergoing the forefoot surgery [30]. In addition to this, another research was conducted in 2019 on considering the predictive score to evaluate the risk of surgical site infection after considering the orthopedic trauma surgery in which the scholars considered the first level trauma patients along with focusing on their 27 factors. In their research, they also found the postoperative infection among the patients and explored the expected risk of pin site infection before the fixation [25]. In the same year, Nick Tellam and others exposed the K-wires-based pin site infection and its related prevention strategies, especially in the pediatric population perspective. According to them, the pin tracks mostly to allow the bacteria to enter and generate an infection. They aimed to majorly explore different ways to overcome the more harmful sequel that is majorly associated with hardware infection. They weekly monitored the post-operative infections by critically reviewing the 782 patients with major fractures of hand. In the end, they concluded that $4 \%$ reduction in the infection rate majorly required early pin removal, oral antibiotics and reoperations.

\section{Treatment Options for Pin Site Infection}

In order to overcome the pin site infection rate, many types of research have already been made to exaggerate this infection problem on the large scale to derive the proper solutions. In 2016, authentic research was conducted by Nikolas Kazmers and others that pin site infections majorly burdened the health system and patient, because such infection increased the number of clinic visits that are required for treatment. Before proposed an authentic treatment, they derived different factors that directly enhanced the pin site infections like the use of prophylactic antibiotics, characteristics of pin design, surgical technique, patient-specific risk factors, and the post-operative pin care protocol i.e. dressing changes, cleaning, and showering. In their secondary data-based research study, scholars majorly recommended different prevention treatment of pin site infection. Their treatment working was associated with pin design i.e. if silver and gold-based pin coating is used in surgery, then its infection rate can be reduced because that directly helped to prevent bacterial adhesive. The second one is associated with a surgical technique that the unicortical placement of wires and pins generates excessive heat that burns the bone which must be avoided. The third one is associated with cleaning solutions that also played a major role to reduce the infection risk like chlorhexidine might be helpful to decrease the colonization of pin site, its antibiotic use, and pain. In addition to this, this research also explored how effective frequency and site cleaning, decreasing type, and showering are useful to overcome the prophylactic antibiotics issues [4]. Somehow similar research has been conducted by other related scholars to exaggerate the treatment of external fixation pins about the wrist by considering 
the randomized trial. According to them, the pin-track infection caused major troublesome complications of external fixation that mostly compromised the successful fracture. In order to critically evaluate the medical condition of patients and proposed their solutions, they majorly considered 180 patients of which 120 of them having wrist issues due to randomize the unstable distal radial fracture. They segregated them into three treatment groups; first one is the weekly dry dressing changes in the absence of pin-site care, the second one is based on daily pin-site care by providing a solution of one-half hydrogen peroxide and one-half normal saline solution, and the third one is treatment along with the placement of chlorhexidine-impregnated discs around the pins. They majorly evaluated their patients in respect to erythema, drainage, cellulitis, radiographic or clinical evidence of pin-loosening, and the need for antibiotics and pin removals before any fracture healing because of infection. In their outcome, 23 patients had a pin-track-based complication in which twelve of them required oral antibiotics for the pin-track infection's treatment. It means no significant difference has existed among these three groups based on the pin-site complications, and the age factor plays a major role to increase the risk of postoperative pin-track complications. They recommended the use of dry and sterile dressing for pin-track care after the external fixation for the distal radial fractures' treatment [31]. In previous scholars, many scholars also worked on exposing the side effects of K-wires in the metacarpal, phalangeal, and distal radical fractures along with exposing the treatment for such infection. To fulfill that aim Ridley Taylor and others majorly conducted a retrospective review by focusing on those patients who are greater than 16 years old. According to their outcomes, those patients who were exposed to K-wires for the fixation of the metacarpal, phalangeal, or distal radius fractures, were more effectively treated for pin-site infection as compared to those whose K-wires buried under the skin. So, metacarpal fractures must be treated with exposed K-wires with two times more likely for a pin-site infection i.e. $8.7 \%$ of buried $\mathrm{K}$-wires cases vs $17.6 \%$ of exposed $\mathrm{K}$ wire cases [32]. In the previous literature, different researches have been made to consider digital surgery to add value to the medical field. According to James Good and Kyle Fiala, the claw toes, hammertoes, and mallet toes are such essential digital deformities that are mostly encountered by ankle and foot surgeons. They stated that mostly digital surgery is majorly rewarded for surgeons and patients that are quite productive than $\mathrm{K}$-wires whose fixation caused different complications and left surgeon to look forward to the best option. In order to derive such advanced treatment based techniques and fixation trends, these scholars majorly considered the physical \& radiographic examination of such infections in forefoot based surgical techniques. They concluded that digital deformities are the best alignment for ankle and foot specialists, because when the conservative treatment failed to eliminate the patient complaint, then digital surgical correction showed a viable treatment option. According to them, such advanced fixation technology and techniques are the best options to make digital 
surgery successful [33]. These scholars significantly proposed the authentic treatment based newer fixation in the forefoot surgery by utilizing some digital surgery techniques that directly help to overcome the usage of K-wires inpatient surgery like Cannulated Digital Fusion Screws, Intramedullary Memory Metal Implant, Memory Staples, and Absorbable Fixation. For example, the aim of providing a maintainable and predictable correction, the use of intramedullary cannulated screws for the PIPJ arthrodesis of the digital deformities' correction provided various advantages in the forms of fixations. Like the risk of pin site infection becomes eliminated and the external hardware is avoided because it maintains the compression across the fusion site that potentially reducing the nonunion's risk. Pin-care protocol is the best treatment way proposed by the previous scholars in their authentic research studies which are mentioned below.

\section{Pin-Care Protocol Based Treatment}

Pin-care protocol is majorly associated with the concept of daily washing and cleaning all the pins and wire sites. This treatment method has already been used by the majority of medical researchers in their researches like Valentin Antoci and others (2008) majorly evaluated the incidence of pin-site infection (PTI) during the process of limb lengthening by using the external fixation in $88 \mathrm{pa}-$ tients. According to their outcomes, the PTI rate was $96.6 \%$ in which rate was significantly higher in half-site fixators as compared to hybrid fixators, so the rate of additional surgeries for treating such kind of surgical infection was much higher for half-pin sites as compared to fine-wire sites; and because of this, three of the 88 cases result in chronic osteomyelitis. In the end, they concluded that such pin site infection rate can be significantly minimized through a careful insertion of well-defined, simple, and excellent pin-care protocol within the surgery [34]. According to them, pin-care protocol mostly involved the sterile gauze packing around the skin-pin junction deeply at the surgery timing and removing it on a postoperative day. According to Jennison, Mcnally, and Pandit, pin site infection is the most effective pin site care regimen to overcome the incidence of pin site infection. They stated that there are many techniques to reduce bacterial adhesion with low toxicity and osteointegration. Like titanium-copper alloys, coatings, chitosan coatings, nanosilver coatings, iodine, chlorhexidine, hydroxyapatite, and antibiotic coatings [35]. Also, Anna Timms majorly worked on considering the guidance and recommendations regarding pin site care that directly help to overcome the pin site infection rate among healthcare professionals, they majorly considered the adults, hospitals, pediatric and community environment of UK to explore the significance of pin site care and its guidance on this surgical area of practices [36]. In the Journal of Injury, Ioannis Ktistakis and others (2015) also worked on considering the importance of pin-site care protocols as an efficient way to reduce the incidence of infection by critically analyzing their effectiveness and outcomes. For that aim, these scholars majorly screened the 369 total manuscripts and only 13 of them met the inclusion crite- 
ria (Table 1) that evaluated the different protocols of pin site care. According to their outcomes, the infection rate was diverse among 574 patients based on the protocol of pin site care and implant type usage. In the end, they concluded that zero percent of infection rate were occurred among patients after using different protocols of pin site care [37]. Also, Anne Lethaby and others considered the significance of pin site care for preventing the infections associated with the external pins and fixators. In their research study, their major aim was to critically explore the different ways of cleaning and dressing the orthopedic percutaneous pin sites. They assessed the independent trials to satisfy the inclusion criteria with extracted data and undertook quality assessment, and at the end concluded that all-around 572 patients based eleven trials were eligible for the inclusion in which three trials were compared with alternatives sterile cleaning solutions, three were compared with cleaning regimen with no cleaning, one was compared the daily pin site care with no care, and the remaining six trials were compared with diverse dressings. The major difference between such trails was majorly based on antibiotics and techniques. They critically evaluated all the surgical outcomes and concluded that the risk of pin site infection was significantly reduced with polyhexamethylene biguanide (PHMB) gauze when it significantly compared to the plain gauze ( $\mathrm{Cl} 0.12$ to 0.44 and $\mathrm{RR} 0.23,95 \%)$. Otherwise, there was no significant difference existed between the groups [35]. The pin site care protocol-based treatment of such infection was also discussed by Jenny Temple and Julie Santy to consider the treatment implication for the prevention of external bone pins and fixation based infection on the human skin. In order to derive a constructive outcome, these scholars majorly considered the randomized controlled trials in the affected people by comparing them with the effect on infection rates of diverse procedures of dressing or cleaning orthopedic percutaneous pin sites. According to their outcomes, only one trial shown the eligible review for the inclusion, when it compared the cleaning with $70 \%$ alcohol, $0.9 \%$ saline, and no cleaning that result in reducing the pin site infection rate [35].

Table 1. Patient inclusion criteria.

\begin{tabular}{cc}
\hline Characteristic & Criteria for Inclusion \\
\hline Age & Between 18 to 44 \\
Gender & Male or Female \\
BMI & Normal, overweight, or obese \\
Allowed Surgeries included & Hammertoe correction, Chevron osteotomy, Lesser MT \\
osteotomy, or the Combined forefoot procedures & Elective forefoot surgeries only \\
Tymber of inserted Pins & Between 25 to 40 days \\
Number of days of Insertion & Yes \\
Allowed diseases & (1 minimum, 4 maximum, \\
\end{tabular}




\section{Use of Antibiotics in Treatment}

As there are various risk factors associated with surgical site infection because of the Pseudomonas, Streptococcus, and Staphylococcus type bacteria. Most of the time, additional surgeries are required to assess and treat the surgical site infections in which antibiotics played a major role. In the journal of pediatric surgery, Johannes Schalamon and others majorly worked on considering the antibiotics treatment based significance to overcome the pin tract infection with the external fixation of pediatric fractures. For that aim, they majorly graded the pic tract infections by using the Dahl classification and obtained the bacterial cultures for the purpose of drainage from pin site. According to their clinical outcomes, the majority infections were moderated or mild and only 3 cases faced severe deep infections. In order to heal that infection, rifamycin was used to heal six, and the cefuroxime and clindamycin based systematic antibiotics were used to treat 35 infections, while the remaining 2 infections need the removal of pin. This shows that majority of infections were managed with specific antibiotics [38]. After this, another related research has been conducted by IIker Uckay and others to develop different authentic ways to prevent surgical site infections, especially for elective clean surgery. In their research, they concluded that there is a need for adequate perioperative administration of the prophylactic antibiotics to overcome the side effects of pin site infection [39]. According to them, the parenteral prophylactic antibiotic agents' choice and the route and timing of administration became standardized in order to develop well-planned prospective clinical studies. In the International Journal of Orthopaedic and Trauma Nursing, Julie Santy majorly worked on reviewing the pin site wound infection assessment criteria by considering the six published sets based secondary study. In the end, they concluded that all the existing tools at that time were flawed in terms of their validity both for research and for practice into the pin site wound care options [40]. Also, Fragomen Austin and others majorly worked on exaggerating the working of prophylactic postoperative antibiotics and its implications that might not significantly reduce the pin site infection after the external fixation. They concluded that there is no clear difference between antibiotics and nonantibiotics treatment groups, especially in the pin site infection case in the postoperative surgical situation. According to them, there is a need to make certain improvements in the surgical instruments and anti-biotics to significantly overcome the infection rate, and oral antibiotics are the best options to resolve such infection rate [41]. This kind of treatment option was also mentioned by Dimitri Ceroni and others in the journal of children's orthopedics where they majorly studied the prevention of pin-tract infection by using the osteomyelitis based treatment during the pediatric external fixation. According to them, external fixator biomechanics and meticulous surgical technique during the pin and wire insertion, postoperative pin removal, and pin site care directly overcome the existence of treatment failures and another infection rate. It shows that physiopathology, epidemiology, and microbiology of the pin-tract infection (PTI) in the 
pediatric populations helped to diagnose, classified, and treat such infections [42]. In addition to this, Suresh Sharma also talked about the empirical evidence of pin site care practices in his research study where he stated that meticulous pin site care played a major role to minimize the infection rate in which the higher variability and inconsistency rate in the pin site care practice played a major role to overcome such skin infection. He majorly used the sterile technique in which the pin sites were cleaned with normal saline, povidone-iodine, hydrogen peroxide, the crust was removed and the pin sites were covered by povidone-iodine soaked gauze, bulky dressing, and dry gauze without applying any ointment that resulted in enhancing the frequency of less infection [43].

\section{Use of Crusts in Treatment}

Crusts can significantly act as a barrier between the insertion site of pin and the external environment in order to significantly overcome the infection rate. Like in 2018, Dimitra-Simone Georgiades majorly worked on exaggerating the systematic integrative review of pin site crusts for the orthopedic clinicians that directly helped them to make evidence-based decisions on the removal of such infections. The goal of such crust based pin-site care was to directly reduce and prevent the pin site infection. They majorly considered the mixed method appraisal tool in order to significantly address the properties of pin site crusts in case of dressing and developing a barrier between the insertion site of pin and the external environment that majorly worked to reduce the infection. They critically evaluated that pin site crusts significantly reduced the pin site infection in the form of a hardened plug that created a physical barrier to bacterial contamination. They also stated such treatment as biological dressing in which the presence of keratino-cytes within the crusts directly sealed the pin sites and acted as a barrier. According to them such crusts directly prevent the organisms from the outside environment to enter the site [44]. But, there were also some limitations in case of lack of understanding regarding the crust retention as a biological dressing with as one clinical trial, lack of systematic review of literature for inclusion, and the lack of heterogeneity among the Britain study based patients. In the authentic journal of injury, Simon Britten and others majorly worked on considering the Ilizarov fixator pin site care by specifically considered the crusts role in the prevention of infection. They specifically considered the rate of infection among two groups of patients based on the fracture stabilization or the lower limb reconstruction by using an Ilizarov fine wire fixator. After critically evaluated both the groups' clinical condition in case of their antibiotics treatment, they concluded that no patient need any hospitalization services for the intravenous antibiotics or any change in the second group, while 3 out of 59 patients from the first group required hospitalization (Table 2). According to their Chi-squared test-based statistical outcomes, they concluded that retention of the adherent crusts during the process of Ilizarov fixator pin site care played a significant role to protect any development of pin site infections, 
but mostly render the infected pin site more refractory to treatment. According to this study outcome, crusts should have remained on the skin as long as the pin site remained uninfected because such retained crusts significantly acted as a physical barrier to bacterial contamination [45]. In addition to this, another similar goal-based research has been made by Ali Turgay Cavusoglu and others to examine the circular external fixation based pin site care by utilizing its two diverse protocols. For that aim, they developed the prospective randomized study for the treatment of tibial fractures along with Ilizarov external fixation as a valuable treatment. They categorized the patients into two major groups and each patient was cleaned by using the sterile gauze impregnated with only ten percent polyvinylpyrrolidone iodine (Polyod) every three days, after this group one was advised to perform pin care by simply daily showering and brushing such pin site through soap and ordinary soft toothbrush; while group two's patients were allowed to perform the pin care by regular showering and cleaning the crust by using the sterile gauze with Polypod. In the end, they concluded that minor infection of all pin sites was resulted in the group one patient with $50.7 \%$ and $43.6 \%$ in group two, while the major infection was determined as $3.7 \%$ in group two and 3.5\% in group one (Table 3). Their outcomes depicted that efficient pin site care could be processed without any impairment of patient comfort and without the prohibition of showing because pin site care could effectively self-managed

Table 2. Percentage of infected patients in light of age group.

\begin{tabular}{cccc}
\hline Gender & Number of Patients & Number of Infected Patients & Percentage of Infected Patients \\
\hline Male & 28 & 13 & $46.42 \%$ \\
Female & 22 & 10 & $45.45 \%$ \\
\hline
\end{tabular}

Table 3. Used prevention techniques details.

\begin{tabular}{ccccc}
\hline & & Frequency & Percent & Valid Percent \\
\hline \multirow{3}{*}{ Valid } & CA & 15 & 30 & 30 \\
& B & 17 & 34 & 34 \\
& PI & 18 & 36 & 36 \\
& Total & 50 & 100.0 & 100.0 \\
\hline
\end{tabular}

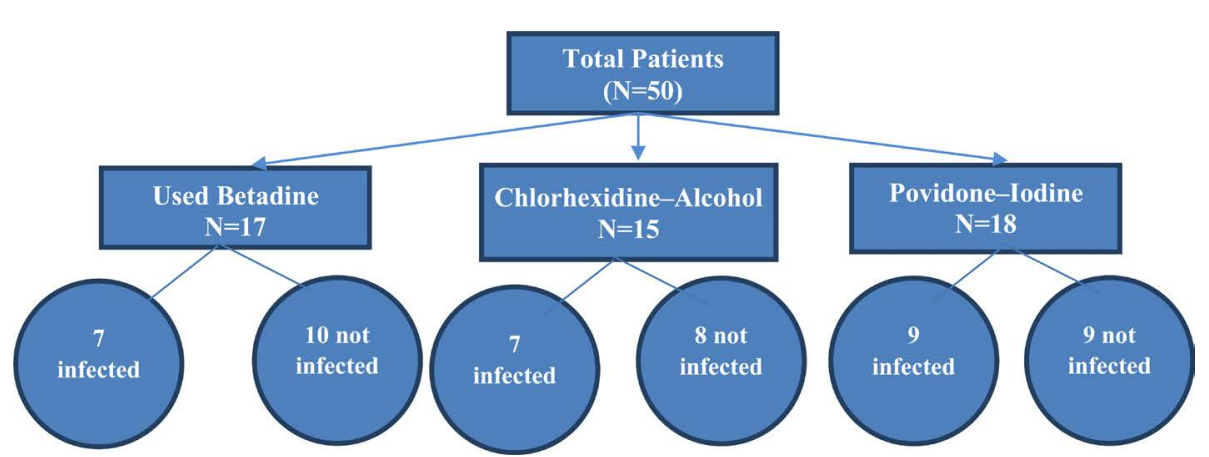

Figure 1. Findings regarding effectiveness of Disinfectant usage. 


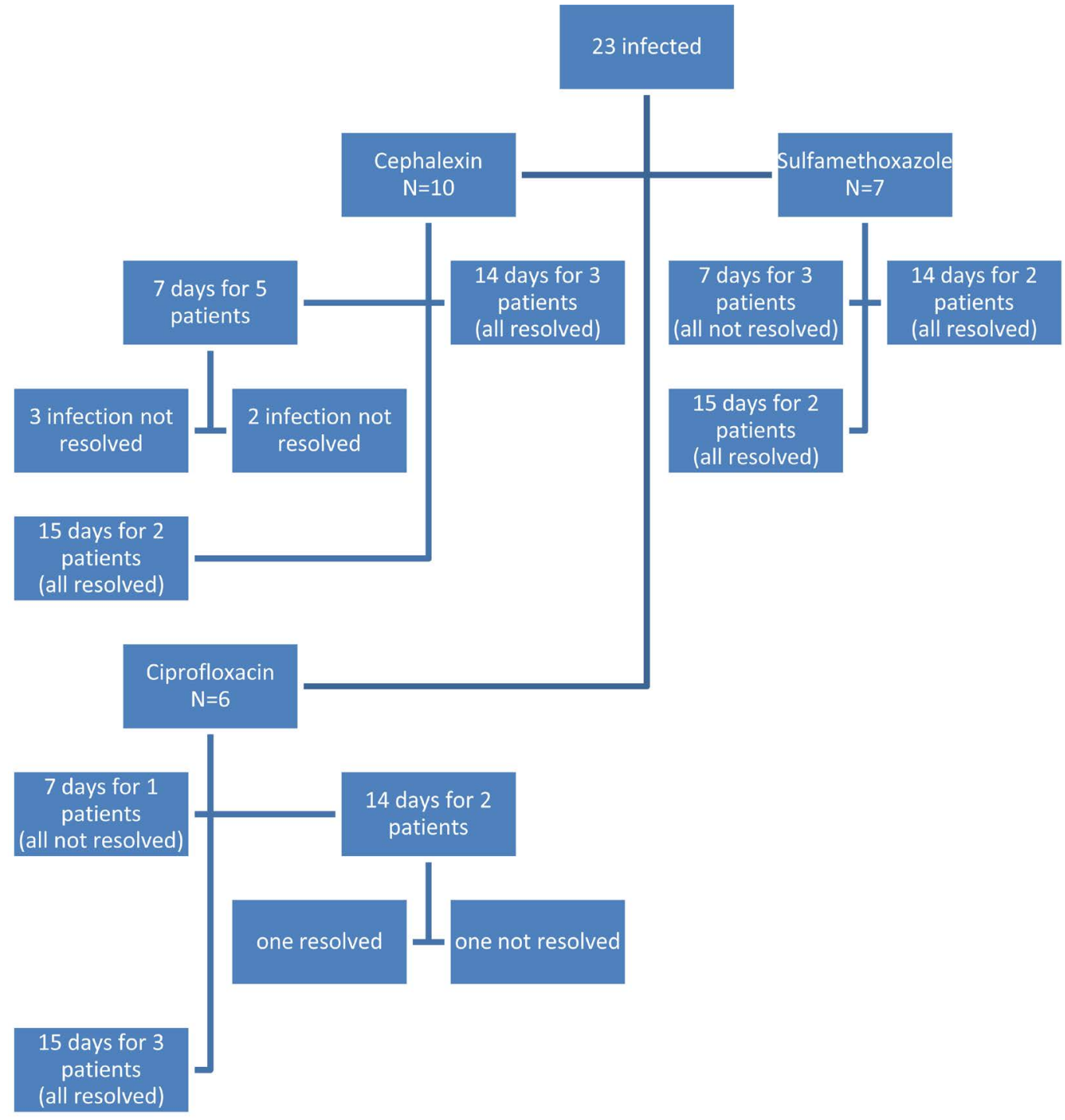

Figure 2. Findings regarding the effectiveness of antibiotic courses.

by the patients as compared to use some complex sterilization techniques [46].

\section{Discussion}

In this study, the data was collected from 50 patients (Figure 1). These data were used to formulate an understanding of the best available treatment methods of preventing and curing pin site infections that occur in the site of k-pin insertions in patients that have undergone elective forefoot surgery with 1 to 4 pins and the cast is removed after 2 weeks. After this removal, a strict prevention treatment was used in which the patients used either of the three disinfectants that are suggested in this study, however (Figure 2), despite using the disinfectants, the infection rate is much higher than the ones reported in past studies in which these disinfectants were used [27] [47] [48] [49]. This can be due to the fact that the patients were advised and sent home to use these prevention techniques instead of proper monitoring of the preventive measures. Moreover, the rate of in- 
fection has been found to be significantly affected (positively) by the factors of BMI, smoking, and surgery type. Unlike past studies, diabetes was not linked to the presence of a higher rate of infection in the patients [32] [50] [51] [52]. Additionally, the antibiotic treatments that have been suggested in this study include Cephalexin, Sulfamethoxazole and Ciprofloxacin. The best treatment is 2 weeks of antibiotics and the most effective, overall, was Cephalexin.

Characteristics of pin design, surgical technique, patient-specific risk factors, and the post-operative pin care protocol i.e. dressing changes, daily washing cleaning, and showering. If silver and gold-based pin coating used in surgery, then its infection rate can be reduced because that directly helped to prevent bacterial adhesive. The second one is associated with a surgical technique that the unicortical placement of wires and pins generates excessive heat that burns the bone which must be avoided. The third one is associated with cleaning solutions that also played a major role to reduce the infection risk like its chlorhexidine might be helpful to decrease the colonization of pin site [4]. There is a need to make certain improvements in the surgical instruments and anti-biotics to significantly overcome the infection rate, and oral antibiotics are the best options to resolve such infection rate [41].

\section{Follow-up}

To conduct this study, the forefoot surgery history of a single surgeon was taken for the past 3 years (2018-2020).

\section{Conclusion}

In this study, the data was collected from 50 patients to collect data regarding preferred techniques used in elective forefoot surgery and the treatments used for infections that may occur. The findings of the study showed that age and diabetes, even neuropathic diabetes, is not linked to the infection rate. However, this disparity from the past results can be due to the fact that the data in this study was limited and the patients that were included were not monitored in a closed environment but instead were sent home with specific prevention instructions. Moreover, the ages of the patients were specifically selected to avoid any interference from complications that can be age-related. In the future, the researchers can include patients that have associated diseases in addition to diabetes such as cardiac diseases, hypertension, and arthritis as these ailments can affect the management techniques of such wounds and their infections.

\section{Summary}

One of the most commonly occurring postoperative syndrome after insertion of k-pins as a fixator is the pin site infection. Previous studies have linked several factors with the occurrence of pin site infection. In this study, regarding patient treatments, the researcher has found that the use of antiseptics as a prevention technique is a good management method for infection, however, it should be conducted in a monitored environment. Moreover, the study has shown that 
Cephalexin is the most effective antibiotic treatment for the infection in forefoot $\mathrm{k}$-pin infections. However, there is a need for further testing and evaluation to deduce more evidence for the disinfection reagents that can help in reducing the occurrence of infections so that the use of antibiotics can be minimized.

\section{Conflicts of Interest}

The authors declare no conflicts of interest regarding the publication of this paper.

\section{References}

[1] Shah, S.D.B.A., Jamil, M., Kabir, S.K., Khan, M.A., Hayat, S. and Muhammad, A. (2018) Intramedullary Kirchner Wires Fixation in Unstable Radius and Ulna Fractures in Children in a Tertiary Care Hospital. Journal of Medical Sciences, 26, 308-311.

[2] Iobst, C., Thompson, R.G., Grauer, J. and Wheeler, P. (2018) How to Prevent K-Wire Bounce in Oblique Supracondylar Humerus Fractures. Journal of Orthopaedic Trauma, 32, e492-e496. https://doi.org/10.1097/BOT.0000000000001273

[3] Van Leeuwen, W.F., van Hoorn, B., Chen, N. and Ring, D. (2016) Kirschner Wire Pin Site Infection in Hand and Wrist Fractures: Incidence Rate and Risk Factors. Journal of Hand Surgery, 41, 990-994. https://doi.org/10.1177/1753193416661280

[4] Kazmers, N.H., Fragomen, A.T. and Rozbruch, S.R. (2016) Prevention of Pin Site Infection in External Fixation: A Review of the Literature. Strategies in Trauma and Limb Reconstruction, 11, 75-85. https://doi.org/10.1007/s11751-016-0256-4

[5] Cancienne, J.M., Cooper, M.T., Laroche, K.A., Verheul, D.W. and Werner, B.C. (2017) Hemoglobin A1c as a Predictor of Postoperative Infection Following Elective Forefoot Surgery. Foot \& Ankle International, 38, 832-837. https://doi.org/10.1177/1071100717705140

[6] Ling, S.K.K., Chan, V., Ho, K., Ling, F. and Liu, T.H. (2018) Reliability and Validity Analysis of the Open-Source Chinese Foot and Ankle Outcome Score (FAOS). Foot, 35, 48-51. https://doi.org/10.1016/j.foot.2017.12.001

[7] Pace, G., Dellenbaugh, S., Stapinski, B., Aydogan, U., Bustillo, J. and Juliano, P. (2017) Antibiotic Use and Kirschner Wire Fixation in Forefoot Surgery: A National Survey. Orthopedics, 40, e594-e597. https://doi.org/10.3928/01477447-20170404-04

[8] McKenzie, J.C., et al. (2019) Incidence and Risk Factors for Pin Site Infection of Exposed Kirschner Wires Following Elective Forefoot Surgery. Foot \& Ankle International, 40, 1154-1159. https://doi.org/10.1177/1071100719855339

[9] Mangwani, J., Gulati, A., Benson, R., Cichero, M. and Williamson, D.M. (2017) Role of Prophylactic Antibiotics in Lesser Toe Fusion Surgery: A Prospective Randomised Controlled Trial. Foot and Ankle Surgery, 23, 50-52. https://doi.org/10.1016/j.fas.2016.02.004

[10] Tang, J., Wu, K., Wang, J. and Zhang, J. (2019) Open Reduction and Compression with Double Kirschner Wires for the Treatment of Old Bony Mallet Finger. Journal of Orthopaedic Surgery and Research, 14, 1-9.

https://doi.org/10.1186/s13018-019-1513-2

[11] Ahmad, I., et al. (2007) Intramedullary Kirchner Wire Fixation of Unstable Radius-Ulna Fractures in Children. Pakistan Journal of Surgery, 23, 209-211.

[12] Vikram, A. (2019) Functional Outcome of Displaced Supracondylar Fracture Hu- 
merus in Children Treated by Closed Reduction and Percutaneous Pinning with Kirchner Wires: An Observational Study. International Journal of Orthopaedics, 5 , 757-761. https://doi.org/10.22271/ortho.2019.v5.i4m.1765

[13] Aladin, A. and Davis, T.R.C. (2005) Dorsal Fracture-Dislocation of the Proximal Interphalangeal Joint: A Comparative Study of Percutaneous Kirschner Wire Fixation versus Open Reduction and Internal Fixation. Journal of Hand Surgery, 30, 120-128. https://doi.org/10.1016/J.JHSB.2004.10.011

[14] Kirkham, S.G. and Millar, M.J. (2012) Cancellous Bone Graft and Kirschner Wire Fixation as a Treatment for Cavitary-Type Scaphoid Nonunions Exhibiting DISI. Hand, 7, 86-93. https://doi.org/10.1007/s11552-011-9375-Z

[15] Koslowsky, T.C., Mader, K., Kirchner, S., Gausepohl, T. and Pennig, D. (2007) Treatment of Medial Malleolar Fractures Using Fine-Threaded K-Wires: A New Operative Technique. The Journal of Trauma and Acute Care Surgery, 62, 258-261. https://doi.org/10.1097/01.ta.0000240443.61205.96

[16] Xie, J., et al. (2018) Kirschner Wire Temporary Intramedullary Fixation Combined with a Locking Anatomical Plate versus a Reconstruction Plate in the Treatment of Comminuted Clavicular Fractures: A Retrospective Study. BioMed Research International, 2018, Article ID: 5017162. https://doi.org/10.1155/2018/5017162

[17] Tosti, R., Foroohar, A., Pizzutillo, P.D. and Herman, M.J. (2015) Kirschner Wire Infections in Pediatric Orthopaedic Surgery. Journal of Pediatric Orthopaedics, 35, 69-73. https://doi.org/10.1097/BPO.0000000000000208

[18] Lakshmanan, P., Dixit, V., Reed, M.R. and Sher, J.L. (2010) Infection Rate of Percutaneous Kirschner Wire Fixation for Distal Radius Fractures. Journal of Orthopaedic Surgery, 18, 85. https://doi.org/10.1177/230949901001800119

[19] Hargreaves, D.G., Drew, S.J. and Eckersley, R. (2004) Kirschner Wire Pin Tract Infection Rates: A Randomized Controlled Trial between Percutaneous and Buried Wires. Journal of Hand Surgery, 29, 374-376. https://doi.org/10.1016/J.JHSB.2004.03.003

[20] Case, A.L., Ty, J.M., Chu, A., Ho, C.A., Bauer, A.S. and Abzug, J.M. (2020) Variation among Surgeons Regarding the Use of Preoperative Antibiotics in Percutaneous Pinning Procedures of the Upper Extremity in the Pediatric Population. Hand, 1558944720944259. https://doi.org/10.1177/1558944720944259

[21] Albright, R.H. and Fleischer, A.E. (2020) Hammertoe Fixation: Traditional Percutaneous Pin versus Internal Fixation. In: Evidence-Based Podiatry, Springer, Berlin, 21-45. https://doi.org/10.1007/978-3-030-50853-1_3

[22] Nery, C. and Baumfeld, D. (2017) Salvage of Lesser Toes Deformities: Revision Forefoot. Techniques in Foot \& Ankle Surgery, 16, 20-27. https://doi.org/10.1097/BTF.0000000000000146

[23] Modha, M.R.K., Morriss-Roberts, C., Smither, M., Larholt, J. and Reilly, I. (2018) Antibiotic Prophylaxis in Foot and Ankle Surgery: A Systematic Review of the Literature. Journal of Foot and Ankle Research, 11, 1-14. https://doi.org/10.1186/s13047-018-0303-0

[24] Tootle, K., Hollnagel, J. and Kinmon, K. (2019) Surgical Treatment of Severe Hallux Abducto Valgus with Dislocation of the First Metatarsophalangeal Joint: A Case Presentation and Surgical Technique. Foot and Ankle Specialist, 12, 281-285. https://doi.org/10.1177/1938640019835300

[25] Wise, B.T., et al. (2019) A Predictive Score for Determining Risk of Surgical Site Infection after Orthopaedic Trauma Surgery. Journal of Orthopaedic Trauma, 33, 506-513. https://doi.org/10.1097/BOT.0000000000001513 
[26] Aragón-Sánchez, J. (2020) Surgery for the Diabetic Foot: Prophylactic and Osteomyelitis Surgery-Is There an Evidence Base? In: The Foot in Diabetes, John Wiley \& Sons Ltd., Hoboken, 375-393. https://doi.org/10.1002/9781119445821.ch23

[27] Hak, D.J., Wiater, P.J., Williams, R.M. and Pierson, C.L. (2005) The Effectiveness of Standard Povidone Iodine Surgical Preparation in Decontaminating External Fixator Components. Injury, 36, 1449-1452. https://doi.org/10.1016/j.injury.2005.05.006

[28] Albright, R.H., et al. (2020) Risk Factors for Failure in Hammertoe Surgery. Foot \& Ankle International, 41, 562-571. https://doi.org/10.1177/1071100720904931

[29] Atinga, M., Dodd, L., Foote, J. and Palmer, S. (2011) Prospective Review of Medium Term Outcomes Following Interpositional Arthroplasty for Hammer Toe Deformity Correction. Foot and Ankle Surgery, 17, 256-258.

https://doi.org/10.1016/j.fas.2010.08.008

[30] Samuel, R., Sloan, A., Patel, K., Aglan, M. and Zubairy, A. (2008) The Efficacy of Combined Popliteal and Ankle Blocks in Forefoot Surgery. JBJS, 90, 1443-1446. https://doi.org/10.2106/JBJS.G.01133

[31] Egol, K.A., Paksima, N., Puopolo, S., Klugman, J., Hiebert, R. and Koval, K.J. (2006) Treatment of External Fixation Pins about the Wrist: A Prospective, Randomized Trial. JBJS, 88, 349-354. https://doi.org/10.2106/00004623-200602000-00014

[32] Ridley, T.J., Freking, W., Erickson, L.O. and Ward, C.M. (2017) Incidence of Treatment for Infection of Buried versus Exposed Kirschner Wires in Phalangeal, Metacarpal, and Distal Radial Fractures. Journal of Hand Surgery, 42, 525-531. https://doi.org/10.1016/j.jhsa.2017.03.040

[33] Good, J. and Fiala, K. (2010) Digital Surgery: Current Trends and Techniques. Clinics in Podiatric Medicine and Surgery, 27, 583-599. https://doi.org/10.1016/j.cpm.2010.06.007

[34] Antoci, V., Ono, C.M., Antoci Jr., V. and Raney, E.M. (2008) Pin-Tract Infection during Limb Lengthening Using External Fixation. The American Journal of Orthopedics (Belle Mead NJ), 37, E150-E1544.

[35] Lethaby, A., Temple, J. and Santy-Tomlinson, J. (2013) Pin Site Care for Preventing Infections Associated with External Bone Fixators and Pins. Cochrane Database Systematic Review, No. 4, CD004551. https://doi.org/10.1002/14651858.CD004551.pub3

[36] Timms, A. and Pugh, H. (2012) Pin Site Care: Guidance and Key Recommendations. Nursing Standard, 27, 50-55. https://doi.org/10.7748/ns2012.09.27.1.50.c9271

[37] Ktistakis, I., Guerado, E. and Giannoudis, P.V. (2015) Pin-Site Care: Can We Reduce the Incidence of Infections? Injury, 46, S35-S39. https://doi.org/10.1016/S0020-1383(15)30009-7

[38] Schalamon, J., Petnehazy, T., Ainoedhofer, H., Zwick, E.B., Singer, G. and Hoellwarth, M.E. (2007) Pin Tract Infection with External Fixation of Pediatric Fractures. Journal of Pediatric Surgery, 42, 1584-1587. https://doi.org/10.1016/j.jpedsurg.2007.04.022

[39] Troadec, M. (2018) Preventing Surgical Site Infections. Oxymag, 31, 8-11. https://doi.org/10.1016/j.oxy.2018.02.003

[40] Santy, J. (2010) A Review of Pin Site Wound Infection Assessment Criteria. International Journal of Orthopaedic and Trauma Nursing, 14, 125-131. https://doi.org/10.1016/j.ijotn.2009.11.002

[41] Fragomen, A.T., Miller, A.O., Brause, B.D., Goldman, V. and Rozbruch, S.R. (2017) 
Prophylactic Postoperative Antibiotics May Not Reduce Pin Site Infections after External Fixation. HSS Journal, 13, 165-170. https://doi.org/10.1007/s11420-016-9539-Z

[42] Ceroni, D., Grumetz, C., Desvachez, O., Pusateri, S., Dunand, P. and Samara, E. (2016) From Prevention of Pin-Tract Infection to Treatment of Osteomyelitis during Paediatric External Fixation. Journal of Children's Orthopaedics, 10, 605-612. https://doi.org/10.1007/s11832-016-0787-8

[43] Sharma, S. and Vati, W. (2006) Empirical Evidences of Pin Site Care Practices. Nursing \& Midwifery Research Journal, 2, 42-49. https://doi.org/10.33698/NRF0049

[44] Georgiades, D.S. (2018) A Systematic Integrative Review of Pin Site Crusts. Orthopaedic Nursing, 37, 36-42. https://doi.org/10.1097/NOR.0000000000000416

[45] Britten, S., Ghoz, A., Duffield, B. and Giannoudis, P.V. (2013) Ilizarov Fixator Pin Site Care: The Role of Crusts in the Prevention of Infection. Injury, 44, 1275-1278. https://doi.org/10.1016/j.injury.2013.07.001

[46] Cavusoglu, A.T., Er, M.S., Inal, S., Ozsoy, M.H., Dincel, V.E. and Sakaogullari, A. (2009) Pin Site Care during Circular External Fixation Using Two Different Protocols. Journal of Orthopaedic Trauma, 23, 724-730. https://doi.org/10.1097/BOT.0b013e3181abbc31

[47] Giannoudis, P.V. and Harwood, P. (2017) Complications after Damage Control Surgery: Pin-Tract Infection. In: Pape, H.-C., et al., Eds., Damage Control Management in the Polytrauma Patient, Springer, Berlin, 297-307.

https://doi.org/10.1007/978-3-319-52429-0_27

[48] Lovy, A.J., Levy, I., Keswani, A. and Hausman, M.R. (2020) Betadine-Soaked Alcohol Pad and Rigid Immobilization: An Inexpensive and Effective Method to Diminish Infections with Exposed Kirschner Wires in Hand Fractures. Plastic Surgery, 28, 83-87. https://doi.org/10.1177/2292550320925924

[49] Ceroni, D., et al. (2007) From Prevention of Pin-Tract Infection to Treatment of Osteomyelitis during Paediatric External Fixation. Journal of Orthopaedic Science, 50, 146-150.

[50] Kramer, W.C., Parman, M. and Marks, R.M. (2015) Hammertoe Correction with k-Wire Fixation. Foot \& Ankle International, 36, 494-502. https://doi.org/10.1177/1071100714568013

[51] Yammine, K. and Assi, C. (2020) A Meta-Analysis of the Types and Outcomes of Conservative Excisional Surgery for Recalcitrant or Infected Diabetic Toe Ulcers. Foot and Ankle Specialist, 13, 152-160. https://doi.org/10.1177/1938640019857795

[52] Hsu, L.P., Schwartz, E.G., Kalainov, D.M., Chen, F. and Makowiec, R.L. (2011) Complications of K-Wire Fixation in Procedures Involving the Hand and Wrist. Journal of Hand Surgery, 36, 610-616. https://doi.org/10.1016/j.jhsa.2011.01.023 